\title{
Ergodic and arithmetical properties of geometrical progression's dynamics and of its orbits
}

\author{
V. Arnold*
}

March 9, 2004

The sequence of the residues of $2^{i}(\bmod n)$ is studied for an odd integer $n$. Its period $T(n)$ is a peculiar function of $n$, and the paper provides many facts on the arithmetical and asymptotical properties of this function and of the corresponding orbit, consisting of $T$ points of $\mathbb{Z} / n \mathbb{Z}$ (generalizing the Fermat et Euler theorems).

The randomness of this orbit is measured by some randomness parameters, comparing the distances distribution of neighboring points of the orbit with the distribution of distances between the neighboring of $T$ randomly chosen elements of $\mathbb{Z} / n \mathbb{Z}$.

The same randomness parameters (showing some kind of neighbors repulsion in geometrical progressions) are also applied to other sequences, like the primes and the arithmetical progression residues, showing the repulsion presence in these cases too.

I would like to thank G. Capitanio for typing this text for me.

\section{Fermat's-Euler's dynamical system}

The Fermat's "small theorem" and its Euler's generalization are essentially applications of the dynamical system theory ideas to arithmetics as it is explained in [1]. The present article describes some new results and conjectures in this young domain of experimental mathematics.

\footnotetext{
*Partially supported by RFBR, grant 02-01-00655.
} 
Fix a natural number $n$. The residues $\bmod n$, relatively prime to $n$ (including 1), form the multiplicative group $\Gamma(n)$. The number of its elements is denoted by the Gauss notation $\varphi(n)$. The function $\varphi$ is called Euler's function and its value is provided by the easy formula

$$
\varphi\left(\prod p_{i}^{a_{i}}\right)=\prod\left[\left(p_{i}-1\right) p_{i}^{a_{i}-1}\right]
$$

where $p_{i}$ are primes. By definition,

$$
\varphi(1)=1, \quad \varphi(p)=p-1, \quad \varphi(15)=8, \quad \varphi(9)=6 .
$$

The Fermat's-Euler's dynamical system acts on $\Gamma(n)$, multiplying its elements by a constant number, $x \mapsto a x$, where the natural number $a$ is relatively prime to $n$.

In the present paper the case $a=2$ will be considered, the number $n$ being odd.

The multiplication by $a$ acts on $\Gamma(n)$ as a permutation of its $\varphi(n)$ elements. This permutation is decomposed into its cycles. The fundamental property of this Fermat's-Euler's permutation is the following (easy) fact:

Fermat's-Euler's Theorem. All the cycles of the Fermat's-Euler's permutation have the same period $T(n)$ (which is related to the cycles number $N(n)$ by the relation $N(n) T(n)=\varphi(n))$.

In other words, the Fermat's-Euler's permutation Young diagram is always rectangular.

Our problem would be to study the functions $T$ and $N$ of $n$, as well as the ergodic asymptotical properties of the orbits of this dynamics, $\left\{2^{i}\right\} \subset$ $\Gamma(n) \subset \mathbb{Z} / n \mathbb{Z}$.

The simplest question is that of the growth rate of the period $T$ with $n$. The growth rate of the Euler's function had been studied already by Gauss, who had essentially deduced the "averaged asymptotics"

$$
\varphi(n) \sim n / \zeta(2)
$$

from the probability $6 / \pi^{2} \approx 2 / 3$ of the "incommensurability" of two random integers.

The "averaged asymptotics" in this formula and in the next ones is the weak asymptotic, defined and discussed for other diophantine problems in 
[2]. Namely, " " is the sign for the convergence statement of the Cesaro's means:

$$
\frac{\sum_{k \leq n} \varphi(k)}{\sum_{k \leq n} k / \zeta(2)} \rightarrow 1 \quad \text { for } n \rightarrow \infty .
$$

The explicit calculation of the few first hundreds of the periods $T(n)$ and of the orbits numbers $N(n)$ (presented in [1]) suggested the "averaged growth" $T \sim n / 3$. (with large fluctuations to both sides, which are, however, infrequent).

We shall now discuss the nonrandomness degree of the set $\left\{2^{i}\right\}$ of the $T$ elements of the orbit in $T(n)$.

Consider first independent choices of $T$ elements from a finite set of $m$ objects (for the $T$ birthdays coincidence problem $m$ being 365). For the random independent choices all the $T$ chosen elements are different in most cases, provided that $T$ is "small" (smaller than $c_{1} \sqrt{m}$ ) and the repetitions occur in most cases, provided that $T$ is "large" (larger than $c_{2} \sqrt{m}$ ). The probabilities smallness is provided in terms of $c_{i}$ by the erf function.

The $T$ orbit's points $\left\{2^{i}(\bmod n),(i=1, \ldots, T)\right\}$ are all different. If the choice of the orbit elements would be the set of random independent choices of $T$ elements from the $m=\varphi(n)$ elements of the group $\Gamma(n)$ (or even from the whole set of the $m=n$ residues $\bmod n$ ), one should therefore expect the average growth rate $T \sim c \sqrt{n}$ (in the weak asymptotic sense) of the time period till the first repetition.

The longer observed periods $(T \sim c n)$ show, it seems, some nonrandomness of the orbit points, which I would call some "repulsion", obstructing the earlier $(T \sim C \sqrt{n})$ repetition of the residues of the elements of a geometrical progression $(\bmod n)$.

This kind of the repulsion deserves a serious study in the frame of the ergodic number theory, and I shall discuss below the first attempts to investigate the randomness degree of the orbit.

If a circle of length 1 is subdivided into $T$ arcs of lengths $x_{i}$ (where $x_{1}+\cdots+x_{T}=1$ ), the sum of the squares of their lengths

$$
A=\sum x_{i}^{2}
$$

might be considered as some measure of the randomness degree of the dividing points in the following way.

For $T$ equal arcs of lengths $1 / T$ one gets the value $A_{0}=1 / T$ (corresponding to the mass concentration at the simplex center). 
For the uniform distribution of the point $x$ along the simplex one gets (after some easy calculations) the averaged value $A_{1}=2 /(T+1)$. The maximal value $A_{2}=1$ is attained for the mass concentrated at the vertices of the simplex (that is for the degenerated arcs, which all have the lengths 0 , except $x_{1}=1$ ).

Thus, the normalized sum of the squares of the lengths, the randomness parameter

$$
R=A / A_{0}=T A,
$$

is a measure of the nonrandomness of the dividing points choices. The purely random choices provide the coefficient value $R_{1}=A_{1} / A_{0}=2 T /(T+1) \approx 2$. The smaller values of $R$ (between the minimal value $R_{0}=1$, attained at the regular arithmetical progression and the purely random choice value $R_{1}$ ) correspond to some kind of mutual repulsion of the dividing points, while the larger values (between the purely random choice value $R_{1} \approx 2$ and the maximal value $R_{2}=T$ ) correspond to the other kind of the nonrandomness

of the dividing points, which might be called their "attraction" (collapsing most arcs to zero). The observed values of the randomness parameter $R$ for the orbits $\left\{2^{i}(\bmod n), i=1, \ldots, T\right\}$ are presented below, in $\S 8$.

\section{Odd numbers classification}

Definition. An odd number $n$ belongs to the class $(N+)$ if the following (Fermat's-Euler's) congruence holds :

$$
2^{\varphi(n) / N} \equiv+1(\bmod n)
$$

It belongs to the class $(M-)$ if holds the congruence

$$
2^{\varphi(n) / M} \equiv-1(\bmod n) .
$$

It follows from the definition that

$$
\begin{aligned}
& (N+) \subset(K+), \text { provided that } K \text { is a divisor of } N ; \\
& (M-) \subset(K-), \text { provided that } M=K(2 m+1) ; \\
& (M-) \subset(K+), \text { provided that } M=K(2 m) .
\end{aligned}
$$


The class $(N+)$ is an ideal in the multiplicative semigroup of the odd natural numbers : for $n \in(N+)$ the multiple $(2 m+1) n$ also belongs to $(N+)$.

I shall next announce some of the facts on these ideals, deduced from the tables of the largest solutions $N(n)$ and $M(n)$ of the congruences (2.1) and (2.2), which I had calculated for the odd values $n<512$ (studying the minimal period).

Some of the observations resulting from these tables are at present proved as theorems, while even more others are still unproved conjectures (some of which I shall also mention below).

I shall describe only a small part of these observations, to do more one should start from the tables of the article [1]. This article contains also the proofs of the following $2+6+7=15$ propositions.

\section{Classes $2 \pm$}

1. Every odd number, divisible by more than one prime, belongs to the class $(2+)$.

2. If $n=p^{a}$ then

$$
\begin{aligned}
& n \in(2+) \quad \text { provided that } p \equiv \pm 1(\bmod 8) \\
& n \in(2-) \quad \text { provided that } p \equiv \pm 3(\bmod 8) \text {.. }
\end{aligned}
$$

Thus each odd number, which is greater than 1 , belongs either to the class $(2+)$, or to the class $(2-)$.

I think, these easy facts should be attributed to Euler (being perhaps known earlier to Fermat and proved by Gauss).

\section{Classes $4 \pm$}

1. Every odd number, divisible by more than two primes, belongs to the class $(4+)$.

Let now $n=p^{a} q^{b}, a>0, b>0$, be the product of the powers of two different primes $p, q$. Then the class of the product $n$ is described by the following 5 statements (see [1] for the proofs):

2. If $p \equiv+1(\bmod 8)$, then $n \in(4+)$. 
3. If $p \equiv q \equiv-1(\bmod 8)$ or $p \equiv q \equiv-3(\bmod 8)$, then $n \in(4+)$.

4. If $p \equiv q \equiv+3(\bmod 8)$, then $n \in(4-)$.

5. If $p \equiv+3(\bmod 8), q \equiv-1$ or $-3(\bmod 8)$, then $n$ is neither in $(4+)$ nor in $(4-): n \bar{\in}((4+) \cup(4-))$.

6. If $p \equiv-3(\bmod 8), q \equiv-1(\bmod 8)$, then $n$ is neither in $(4+)$ nor in $(4-): n \bar{\epsilon}((4+) \cup(4-))$.

The combinations of the residues cover all the cases, and the following table might help to find the classes of the products from the residues of the primes $(\bmod 8)$.

\begin{tabular}{|c|c|c|c|c|}
\hline$q(\bmod 8)^{p(\bmod 8)}$ & 1 & 3 & 5 & 7 \\
\hline 1 & + & + & + & + \\
\hline 3 & + & - & 0 & 0 \\
\hline 5 & + & 0 & + & 0 \\
\hline 7 & + & 0 & 0 & + \\
\hline
\end{tabular}

In this matrix $5 \equiv-3(\bmod 8), 7 \equiv-1(\bmod 8)$ and the symbol "+" means " $n \in(4+)$ ", while the symbol "-" means " $n \in(4-)$ ", the meaning of the symbol " 0 " being $n \bar{\in}((4+) \cup(4-))$, that is "no such product belongs to the class $(4+)$ and to the class $(4-)$.

It is also useful to quote from [1] the following 7 facts.

7. No $n \equiv-3(\bmod 8)$ and no $n \equiv-1(\bmod 8)$ belong to the class $(8-)$.

8. If $n=p^{a}$ ( $p$ being a prime) belongs to the class $(4+) \cup(4-)$, then $p \equiv 1(\bmod 8)$.

9. The intersection of the classes $(K+)$ and $(N+)$ is the class $(X+), X$ being the smallest common multiple of the numbers $K$ and $N$.

10. The class $(M-)$ is void if $M$ is odd.

11. The classes $(2-)$ and $(N+)$ do intersect exactly along the class $(2 N-)$, if $N$ is odd and do not intersect each other if $N$ is even. 
12. The numbers of the class (6-) are the powers of the primes congruent to $\pm 3(\bmod 8)$.

13. The primes of the class $(6+)$ are congruent to $\pm 1(\bmod 8)$.

\section{The classes $\left(2^{k}+\right)$}

I shall start from the following continuation of the preceding proposition 1 on the class $(4+)$.

Theorem 1. Every odd number, divided by more than $k$ different primes, belongs to the class $\left(2^{k}+\right)$.

Proof. The case $k=1$ and 2 are already discussed in [1] (see 1 in $\S 2$ above). We shall deduce Theorem 1 for the $k+1$ divisors case, supposing it to be already known for the case of $k$ divisors. Denote the product of the powers of $k+1$ different primes by $n=p^{a} R q^{b}, a>0, b>0, R$ containing $k-1$ different primes all unequals to $p$ and to $q$.

The Euler function (evident) multiplicativity provides the identity

$$
\varphi(n)=\varphi(A) \varphi\left(q^{b}\right)=\varphi\left(p^{a}\right) \varphi(B), \quad \text { where } A=p^{a} R, B=R q^{b} .
$$

The numbers $A$ and $B$ having $k$ different prime divisors each, the induction assumption provides the integers $U$ and $V$, such that

$$
2^{\varphi(A) / 2^{k}}=1+A U, \quad 2^{\varphi(B) / 2^{k}}=1+B V .
$$

The numbers $\varphi\left(q^{b}\right)=2 r$ and $\varphi\left(p^{a}\right)=2 s$ being even ( $p$ and $q$ being odd primes) we deduce the representations (with integer coefficients $U^{\prime}, V^{\prime}$ ) :

$$
\begin{aligned}
& 2^{\varphi(n) / 2^{k+1}}=\left(2^{\varphi(A) / 2^{k}}\right)^{r}=(1+A U)^{r}=1+A U^{\prime}, \\
& 2^{\varphi(n) / 2^{k+1}}=\left(2^{\varphi(B) / 2^{k}}\right)^{s}=(1+B V)^{s}=1+B V^{\prime} .
\end{aligned}
$$

The equality of both expressions for the left hand side,

$$
1+A U^{\prime}=1+B V^{\prime},
$$

provides the divisibility of the coefficient $U^{\prime}$ by the factor $q^{b}$ of $B: U^{\prime}=C q^{b}$. Hence the number $n$ belongs to the class $\left(2^{k+1}+\right)$ :

$$
2^{\varphi(n) / 2^{k+1}}=1+C p^{a} R q^{b} \equiv 1(\bmod n) .
$$


Remark. It is easy to find the examples, where $N(n)=2^{k}$ in the situation of Theorem 1 (and in particular, these $n$ do not belong to the class $\left(2^{k+1}+\right.$ ): $n=15$ for $k=2, n=105$ for $k=3, n=3 \cdot 5 \cdot 7 \cdot 11$ for $k=4$.

To obtain the examples with more prime factors one might take the product $n=\prod p_{i}$, where each prime has the form $p_{i}=2 q_{i}+1, q_{i}$ being prime.

To make the number of factors arbitrary large, one should know that the number of the pairs $(q, p=2 q+1)$ of primes is infinite.

The following euristical probabilistic arguments provide some foundation for this statement. The "probability" for $q$ to be prime is (for large $q$ ) of the Chebyshev order $1 /(\ln q)$. Considering the events " $q$ is prime" and $p=2 q+1$ is prime" to be "independent", one evaluates the "probability" for the pair $(q, 2 q+1)$ to be a pair of primes of the order $1 /(\ln q)(\ln (2 q+1))$. The divergence of the "probabilities" series imply the "almost certain" occurrence of an infinite sequence of events by the general probability theory laws.

Of course, the foundation of this euristical "probabilistic reasoning" (which provides also the arguments for the gemini problem) is not easy (the "independence" evaluation requiring some kind of Riemann conjecture).

\section{Triple products of the classes (8+) and (8-)}

If an odd integer have more than 3 different prime divisors, it belongs to the class $(8+)$ by Theorem 1 of section $\S 3$. We shall study now the cases of 3,2 and 1 primes.

Case $n=p^{a} q^{b} r^{c}, a>0, b>0, c>0$.

Denote by I, II, III, IV the sets of the primes, congruent to $1,3,5$ and to $7(\bmod 8)$ respectively. We shall call these primes the primes of the kinds $\mathrm{I}$, II, III, IV.

Theorem 2. The triple product $n$ belongs to the class $(8+)$, provided that the three primes $p, q$ and $r$ do belong to one of the following 7 triples of kinds:

(I, I, X), (I, III, X), (III, III, X), (I, II, II), (I, II, IV), (I, IV, IV), (IV, IV, IV) .

Theorem 3. The triple product $n$ belongs to the class (8-), whenever the primes $(p, q, r)$ do all belong to the kind II.

Theorem 4. No triple product $n$ belongs to the class $(8+)$ or $(8-)$, provided that the primes $(p, q, r)$ kinds triple is one of the 5 triples

$$
\text { (III, II, II), (III, II, IV), (III, IV, IV), (II, IV, II), (II, IV, IV) . }
$$


Remark. The unordered kinds lists of the Theorems 2-4 cover (taking the permutations into account) all the 64 possible ordered lists of the 3 kinds of $p, q$ and $r$.

To verify it, one counts the permuted versions (avoiding to repeat those cases which had already by counted). Listing the versions in the order of the cases of the Theorems 2, 3 and 4, we get the following numbers of the ordered kinds triples :

Theorem $2: 1+3+3+3=10,0+6+3+6=15,0+3+1+3=7$ cases of the first three triples of kinds, and 3, 6, 3 and 1 cases for for the next four triples, making the total number of cases equal to $32+13=45$.

Theorem $3: 1$ case.

Theorem 4 : the 5 triples provide respectively 3, 6, 3, 3, 3 cases, making 18 cases the total number.

Thus the total number of the cases in all the three Theorems is $45+1+$ $18=64$, covering all the ordered kinds possibilities.

Proofs of Theorems 2-4. The multiplicativity property of the Euler function provides the 3 fractions representation,

$$
\frac{\varphi(n)}{8}=\frac{\varphi\left(p^{a}\right)}{2} \frac{\varphi\left(q^{b}\right)}{2} \frac{\varphi\left(r^{c}\right)}{2}
$$

The fraction $\varphi\left(p^{a}\right) / 2=p^{a}(p-1) / 2$ is an even number for the primes $p$ of the kinds I or III and is odd for the prime $p$ of the kind II and IV. If this fraction is even, we get the even product $\left(\varphi\left(p^{a}\right) / 2\right)\left(\varphi\left(q^{b}\right) / 2\right)=2 m$, whence the representation (with integer coefficients $A, A^{\prime}$ )

$$
2^{\varphi(n) / 8}=\left(2^{\frac{\varphi\left(r^{c}\right)}{2}}\right)^{2 m}=\left( \pm 1+A r^{c}\right)^{2 m}=1+A^{\prime} r^{c}
$$

making it impossible for $n$ to belong to the class (8-), along which class holds the representation (with an integer coefficient $B$ )

$$
2^{\varphi(n) / 8}=-1+r^{c}\left(p^{a} q^{b}\right) B
$$

The case $(8-)$ is therefore excluded if there is an even fraction.

We shall see now, whether $n$ belongs to the class (8+).

If there are two even fractions among the three, then we find for each of the three primes (say, for $p_{i}$ ) that the product of the two others fractions 
is even (say, being equal to $2 m$ ). In this case the preceding calculations $(r$ being replaced by $p_{i}$ ) would provide the three congruences (with integer $A_{i}$ )

$$
2^{\varphi(n) / 8}=1+A_{i} p_{i}^{a_{i}} .
$$

The equality of these 3 representations of the same left hand side number implies the divisibility of the coefficient $A_{i}$ by the product $\prod p_{j}^{a_{j}}$ of the missing primes, therefore we get the integer $C$ representation

$$
2^{\varphi(n) / 8}=1+C n, \quad n \in(8+) .
$$

We have thus proved that $n$ belongs to the class $(8+)$ in the first three cases of Theorem 2. tion

If there is only one even fraction among the three, we get the representa-

$$
\frac{\varphi(n)}{8}=\left(\frac{\varphi\left(p^{a}\right)}{2}\right)(2 m+1) .
$$

Therefore, from the $(2+)$ congruence of $\S 2$ (classes $(2 \pm)$ description) we get the representation

$$
2^{\varphi\left(p^{a}\right) / 2}=\left(\alpha+A p^{a}\right)^{2 m+1}=\alpha+A^{\prime} p^{a},
$$

with the same sign $\alpha$ ( $A$ and $A^{\prime}$ being integers).

For the belonging of the number $n$ to the class $(8+)$ one needs the equality $\alpha=+1$, that is $p^{a} \in(2+)$, which means $p \in($ IUIV). Therefore, if $p \in(\mathrm{I} \cup I I I)$, $q$ and $r$ being in (II $\cup \mathrm{IV}$ ), we deduce that $n \in(8+)$ if $p \in \mathrm{I}$, while for $p \in \mathrm{III}$, $n$ can't belong neither to (8+) nor (8-).

We have thus proved the statements $4,5,6$ of Theorem 2 and the statements 1, 2, and 3 of Theorem 4 .

In the remaining cases all the three fractions $\varphi\left(p_{i}^{a_{i}}\right) / 2$ are odd, that is all the primes $p_{i}$ belong to the kinds II $\cup \mathrm{IV}$, we get the (integer coefficients $A_{i}$ ) representations

$$
2^{\varphi\left(p_{i}^{a_{i}}\right) / 2}=\alpha\left(p_{i}\right)+A_{i} p_{i}^{a_{i}},
$$

where $\alpha\left(p_{i}\right)=+1$ for $p \in \mathrm{IV}, \alpha\left(p_{i}\right)=-1$ for $p \in$ II (accordingly to the statement on the classes $(2 \pm)$ of $\S 2)$.

Moreover, the product $\prod\left(\varphi\left(p_{j}^{a_{j}}\right) / 2\right)$ (where $j \neq i$ ) is odd, therefore we get the (integer coefficients $A_{i}^{\prime}$ ) representation

$$
2^{\varphi(n) / 8}=\left(2^{\varphi\left(p_{i}^{a_{i}}\right) / 2}\right)^{2 m_{i}+1}=\alpha\left(p_{i}\right)+A_{i}^{\prime} p_{i}^{a_{i}} .
$$


Hence all the three numbers $\alpha\left(p_{i}\right)$ should be equal to +1 , if $n$ belongs to $(8+)$, and should be -1 , if $n$ belongs to (8-), while if both values \pm 1 are presented, the number $n$ does not belong neither to $(8+)$ nor $(8-)$.

Therefore, the triple of the kinds (II, II, II) provides $n \in(8-)$, the triple $(\mathrm{IV}, \mathrm{IV}, \mathrm{IV})$ the belonging of $n$ to the class $(8+)$, and the presence of both kinds II and IV makes it impossible for $n$ to belong to the union of the classes $(8+) \cup(8-)$.

This conclusion ends the proofs of the Theorems 2-4.

\section{Binary products belonging to class $(8+)$ and $(8-)$}

We consider now the product $n=p^{a} q^{b}, a>0, b>0$, where $p$ and $q$ are different odd primes.

Theorem 5. The product $n=p^{a} q^{b}$ belongs to the class $(8+)$, provided that $p \in \mathrm{I}$ and $q \in(\mathrm{I} \cup \mathrm{III})$.

Theorem 6. If $p$ and $q$ belong to the union (II $\cup \mathrm{IV})$, the product $n=p^{a} q^{b}$ does not belong to the union of classes $(8+) \cup(8-)$.

Theorem 7. If $p$ and $q$ are both congruent to $5(\bmod 8),(p \in \mathrm{III}, q \in \mathrm{III})$, the product $p^{a} q^{b}$ belong to the class (8-).

Theorem 8. If $p \in(\mathrm{I} \cap(4+)), q \in(\mathrm{II} \cup \mathrm{IV})$, then the product $n=p^{a} q^{b}$ belongs to the classe $(8+)$.

Theorem 9. If $p \in(\mathrm{I} \cap(4-)), q \in(\mathrm{II} \cup \mathrm{IV})$, then the product $n=p^{a} q^{b}$ does not belong to the union of classes $(8+) \cup(8-)$.

Remark. Any prime $p$ of kind I $(p \equiv 1(\bmod 8))$ belongs either to the class $(4+)$, or to the class $(4-)$. This remark implies that Theorems 5-9 do cover all the possibilities. The resulting products classes are listed in the following table: 


\begin{tabular}{||c||c|c||c|c|c||}
\hline \hline $\mathrm{q}$ & $\mathrm{I} \cap(4+)$ & $\mathrm{I} \cap(4-)$ & $\mathrm{II}$ & $\mathrm{III}$ & $\mathrm{IV}$ \\
\hline \hline $\mathrm{I} \cap(4+)$ & $(8+)$ & $(8+)$ & $(8+)$ & $(8+)$ & $(8+)$ \\
\hline $\mathrm{I} \cap(4-)$ & $(8+)$ & $(8+)$ & 0 & $(8+)$ & 0 \\
\hline \hline $\mathrm{II}$ & $(8+)$ & 0 & 0 & 0 & 0 \\
\hline $\mathrm{III}$ & $(8+)$ & $(8+)$ & 0 & $(8-)$ & 0 \\
\hline $\mathrm{IV}$ & $(8+)$ & 0 & 0 & 0 & 0 \\
\hline \hline
\end{tabular}

In this table the symbols " $8+$ " and " $8-$ " mean " $\left(n=p^{a} q^{b}\right) \in(8+)$ " and " $\left(n=p^{a} q^{b}\right) \in(8-)$ ", while " 0 " means " $n=p^{a} q^{b}$ never belongs to the union of the classes (8+) and (8-)".

Proof of the Remark. By the $\S 3$ results, $p \in(2+)$, whence the (integer coefficient $A$ ) representation

$$
2^{\varphi(p) / 2}-1=\left(2^{\varphi(p) / 4}-1\right)\left(2^{\varphi(p) / 4}+1\right)=A p .
$$

Hence one of the brackets is divisible by the prime number $p$, whence $p \in$ $((4+) \cup(4-))$.

Proof of Theorem 6. If $n \in((8+) \cup(8-))$, the number

$$
\varphi(n)=(p-1)(q-1) p^{a-1} q^{b-1}
$$

is divisible by 8 , hence at least one of the factors $p-1, q-1$ is divisible by 4 , therefore either $p$ or $q$ belongs to $\{4 s+1\}=\mathrm{I} \cup \mathrm{III}$.

Proof of Theorem 5. If $p \in \mathrm{I}, q \in \mathrm{I} \cup \mathrm{III}$, then numbers $p-1$ and $q-1$ are divisible by 8 and by 4 , hence both numbers $\varphi\left(p^{a}\right) / 4$ and $\varphi\left(q^{b}\right) / 2$ are even. By statement 2 of $\S 2, p^{a} \in(2+), q^{b} \in(2+)$ or $(2-)$ :

$$
2^{\varphi\left(p^{a}\right) / 2}=1+A p^{a}, \quad 2^{\varphi\left(q^{b}\right) / 2}= \pm 1+A q^{b} .
$$

Therefore we get the representations (with integers $A^{\prime}$ and $B^{\prime}$ )

$$
\begin{aligned}
& 2^{\varphi(n) / 8}=\left(2^{\varphi\left(p^{a}\right) / 2}\right)^{\varphi\left(q^{b}\right) / 4}=\left(1+A p^{a}\right)^{\varphi\left(q^{b}\right) / 4}=1+A^{\prime} p^{a} \\
& 2^{\varphi(n) / 8}=\left(2^{\varphi\left(q^{b}\right) / 2}\right)^{\varphi\left(p^{a}\right) / 4}=\left( \pm 1+B q^{b}\right)^{\varphi\left(p^{a}\right) / 4}=1+B^{\prime} q^{b}
\end{aligned}
$$

(the \pm disappearing, the fraction $\varphi\left(p^{a}\right) / 4$ being even for $p \in \mathrm{I}$ ). 
The equality of both representation of the left hand side number implies the identity $1+A^{\prime} p^{a}=1+B^{\prime} q^{b}$, therefore $A^{\prime}=C q^{b}$, whence the (integer coefficient $C$ ) representation

$$
2^{\varphi(n) / 8}=1+C p^{a} q^{b}, \quad n \in(8+)
$$

and Theorem 5 is proven.

Proof of Theorem 7. Now $p, q \in$ III, therefore both fractions $\varphi\left(p^{a}\right) / 4$ and $\varphi\left(q^{b}\right) / 4$ are odd numbers, while both numbers $p^{a}$ and $q^{b}$ belong to the class $(2-)$ : we have the (integer coefficients $A, B, A^{\prime}, B^{\prime}$ ) representations

$$
\begin{gathered}
2^{\varphi\left(p^{a}\right) / 2}=-1+A p^{a}, \quad 2^{\varphi\left(b^{q}\right) / 2}=-1+B q^{b}, \\
2^{\varphi(n) / 8}=\left(-1+A p^{a}\right)^{2 m+1}=-1+A^{\prime} p^{a}, \\
2^{\varphi(n) / 8}=\left(-1+B q^{b}\right)^{2 r+1}=-1+B^{\prime} q^{b},
\end{gathered}
$$

whence the divisibility $A^{\prime}=C q^{b}$ and therefore the (integer coefficient $C$ ) representation

$$
2^{\varphi(n) / 8}=-1+C p^{a} q^{b}
$$

proving the Theorem 7: $n \in(8-)$.

Proof of Theorems 8 and 9. In these Theorems $p=8 c+1, q=4 d+3$, therefore the number $\varphi\left(p^{a}\right)$ is divisible by 8 , the number $m=\varphi\left(q^{b}\right) / 2$ being odd.

The conditions $p \in(4 \pm)$ of these Theorems mean the representation (with integer coefficient $A$ )

$$
2^{\varphi(p) / 4}=\alpha+A p
$$

where $\alpha= \pm 1$ (depending of the sign of the class $(4+)$ ).

Using the relation $\varphi\left(p^{a}\right)=p^{a-1} \varphi(p)$ and iterating the odd degree $p$ calculation of both sides of the preceeding representation several times, we deduce the representation (with integer coefficient $A^{\prime}$ )

$$
2^{\varphi\left(p^{a}\right) / 4}=\alpha+A^{\prime} p^{a}
$$

(preserving the value of $\alpha, p$ being odd).

Therefore we get the (integer coefficient $A^{\prime}$ ) representation

$$
2^{\varphi(n) / 8}=\left(2^{\varphi\left(p^{a}\right) / 4}\right)^{\varphi\left(q^{b}\right) / 2}=\left(\alpha+A^{\prime} p^{a}\right)^{m} .
$$


The number $m=\varphi\left(q^{b}\right) / 2$ is odd (since $q=4 d+3$ ), therefore finally we obtain (with the same $\alpha$ ) the (integer coefficient $A^{\prime \prime}$ ) representation

$$
2^{\varphi(n) / 8}=\alpha+A^{\prime \prime} p^{a} .
$$

At the same time we have another representation, with an integer $B$,

$$
2^{\varphi(n) / 8}=\left(2^{\varphi\left(q^{b}\right) / 2}\right)^{\varphi\left(p^{a}\right) / 4}=\left(\beta+B q^{b}\right)^{r}
$$

where $\beta= \pm 1$, the number $r$ being even, since $p \equiv 1(\bmod 8)$. Its eveness implies the representation (with an integer coefficient $B^{\prime}$ )

$$
2^{\varphi(n) / 8}=1+B^{\prime} q^{b} .
$$

The above two representations of the left hand side number imply that in the case $\alpha=1$ the number $A^{\prime \prime}$ is divisible by $q^{b}$, and therefore we get an (integer coefficient $C$ ) representation

$$
2^{\varphi(n) / 8}=1+C p^{a} q^{b}
$$

whence $n \in(8+)$, and Theorem 8 is proven.

In the opposite case, when $\alpha=-1$, we would deduce from $n \in(8+)$ the two representations (with integer coefficients $A$ and $A^{\prime \prime}$ )

$$
\begin{aligned}
& 2^{\varphi(n) / 8}=+1+A p^{a} q^{b}, \\
& 2^{\varphi(n) / 8}=-1+A^{\prime \prime} p^{a},
\end{aligned}
$$

implying the divisibility of 2 by the odd number $p^{a}>1$.

Similarly, from $n \in(8-)$ we would deduce the two representations (with integer coefficients $A$ and $B^{\prime}$ )

$$
\begin{gathered}
2^{\varphi(n) / 8}=-1+A p^{a} q^{b} \\
2^{\varphi(n) / 8}=+1+B^{\prime} q^{b}
\end{gathered}
$$

implying the divisibility of 2 by the odd number $q^{b}>1$.

The primes $p$ and $q$ being odd, these divisibilities are impossible, hence $n \bar{\in}((8+) \cup(8-))$, and Theorem 9 is thus proven. 


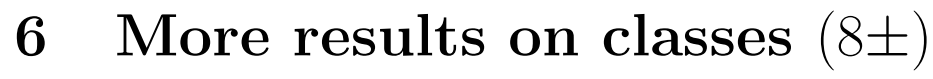

Theorem 10. The product pq of two primes belongs to the class (8+), provided that every prime belongs to the union of classes (4+) and (4-).

Proof. It follows from the property 8 of the classes $(4 \pm)$, mentioned in $\S 2$, that $p \in \mathrm{I}, q \in \mathrm{I}$. Therefore, Theorem 5 implies the belonging of the product to the class $(8+)$. Theorem 10 is thus proven.

Theorem 11. There are no numbers of class (8-) among the numbers congruent to $7(\bmod 8)$.

Proof. If the number of the different prime divisors of our number $n$ is greater then 3 , then $n \in(8+)$ by Theorem 1 of $\S 3$ and therefore it does not belong to the class $(8-)$.

For $n=p^{a} q^{b} r^{c}$ to be of class (8-), each of the three primes $(p, q, r)$ would be congruent to $-3(\bmod 8)$ by Theorems $2-4$ above. The product $n$ is therefore congruent either to 1 (if the multiplicities sum $a+b+c$ is even), or to -3 (if it is odd). Therefore $n$ cannot be congruent to $7(\bmod 8)$.

For $n=p^{a} q^{b}$ to be of class (8-), each of the two primes $p$ and $q$ should be congruent to $-3(\bmod 8)$ (by Theorems $5-9$ above). In this case $n$ is also congruent $(\bmod 8)$ either to 1 (provided that $a+b$ is even) or to -3 (provided that it is odd), and therefore $n$ cannot be congruent to $7(\bmod 8)$.

Finally, the number $n=p^{a}$ cannot be congruent to $7(\bmod 8)$, since $\varphi(n)$ is not divisible by 8 for $p$ congruent to $7(\bmod 8)$.

Theorem 11 is therefore proven.

\section{On the quadratic residues of the primes of the ideal $(8+)$}

The calculations of the prime generators of the ideals $(N+)$, made in [1], showed the following strange phenomenon : their residues $\left(\bmod N^{2}\right)$ are quadratic residues!

While this numerical observation (made in [1] for $N=3$ and 5 but failing for $N=6$ and 9) has no theoretical explanations. I had also calculated the residues $(\bmod 64)$ of the first primes of the class $(8+)$.

These primes' list starts with the numbers

$$
(73,89,233,257,337,601,881,973) \text {. }
$$


Their residues $(\bmod 64)$ are

$$
(9,25,41,1,17,25,49,41) \text {. }
$$

The quadratic residues (mod 64) are

$$
\{0,1,4,9,16,25,36,49,0,17,36,57,16,41,4,33\} \text {. }
$$

The numbers $n$ of class $(8+)$ being odd and 64 being even, the residues of $n(\bmod 64)$ should be odd, and the list of the residues of the primes of class $(8+)$ contains exactly all the odd quadratic residues (mod 64$)$ : it follows from the above list completed by the next primes in $(8+)$,

$$
(1217,1249,1289,1433,1553,1609,1721,1777)
$$

whose residues

$$
(1,33,9,25,17,9,57,49)
$$

include the missing quadratic residue 57 of the starting list.

I am grateful to F. Aicardi for the calculation of these completing generators and residues.

\section{Regularity parameter value}

We consider the residues $(\bmod n)$ as points on the circle of length one, normalising the squared difference sum, dividing it by $n^{2}$ :

$$
\tilde{A}(\{x\})=\sum\left(z_{i}-z_{i+1}\right)^{2} / n^{2}, \quad i=1, \ldots, T
$$

$z_{i} \in \mathbb{Z} / n \mathbb{Z}$. The residues $z_{i}$ are represented here by the ordered numbers from 1 to $n, 1 \leq z_{1}<z_{2}<\ldots<z_{T} \leq n$.

The randomness parameter $R$ of the set $\left\{z_{i}\right\}$ of $T$ points is then defined as the ratio of $\tilde{A}$ to $\tilde{A}_{0}=1 / T$ (the 0 corresponding to the regular sequence $\left.z_{i}=i / n \in \mathbb{R} / n \mathbb{R}\right): R=T \tilde{A}$.

We also consider the distributions of $\bmod n$ residues set $s$ along the group $\Gamma(n)$ (of the $\varphi(n)$ residues, relatively prime to $n$ ). In this case the coordinate $z$ of a residue $r$ is its index in the sequence of ordered residues, relatively prime to $n$ :

$$
1=r_{1}<r_{2}<r_{3}<\ldots<r_{\varphi(n)}<n
$$


Therefore, studying a set of $T$ such residues (say, provided by the orbit $\left\{2^{i}\right\}$ ), we represent them in the form

$$
1 \leq r_{z_{1}}<r_{z_{2}}<\ldots<r_{z_{T}}<n, \quad, z_{T+1}=z_{1},
$$

and use to evaluate the $\Gamma$-randomness parameter $R_{\gamma}=T \tilde{A}_{\Gamma}$, where

$$
\tilde{A}_{\Gamma}\left(\left\{r_{z_{i}}\right\}\right)=\sum\left(z_{i}-z_{i+1}\right)^{2} / \varphi(n)^{2}, \quad(i=1, \ldots, T) .
$$

This $\Gamma$-randomness parameter $R_{\Gamma}$ of a set characterizes the randomness degree of the distribution of $T$ points of the subset of $\Gamma(n)$ among the $\varphi(n)$ points of $\Gamma(n)$. It might differ from the randomness parameter value $R$ for the same set of residues inside the whole group $\mathbb{Z}_{n}=\mathbb{Z} / n \mathbb{Z}$ in the case, when the subgroup $\Gamma(n)$ is badly distributed in $\mathbb{Z}_{n}$.

The observed values for the geometric progression $\left\{2^{i}\right\}$ orbits in $\mathbb{Z}_{n}$ and in $\Gamma(n)$ are represented in the following table, calculated by F. Aicardi on the base of [1]. The numbers in the upper line of the table are proportional to the quantities of those odd numbers, $1<n \leq 511$, for which the values of the randomness parameter of $\left\{2^{i}\right\}$ belong to the interval between the second and the third lines values.

The first line fractions are normalized, dividing the convenient odd numbers quantities by the total number of the values of $n$ (which is 255). The sum of these fractions, approximately 0.86 , shows that for approximately $14 \%$ of the numbers $1<n \leq 511$ the randomness parameter $R$ of the geometrical progression $\left\{2^{i}\right\}$ is greater than 14 .

\begin{tabular}{||c|c|c|c|c|c|c|c||}
\hline \hline Fraction & 0.504 & 0.122 & 0.059 & 0.027 & 0.031 & 0.016 & 0.016 \\
\hline$R_{\min }$ & 1 & 2 & 3 & 4 & 5 & 6 & 7 \\
\hline$R_{\max }$ & 2 & 3 & 4 & 5 & 6 & 7 & 8 \\
\hline \hline
\end{tabular}

\begin{tabular}{||c|c|c|c|c|c|c||}
\hline \hline Fraction & 0.016 & 0.016 & 0.019 & 0.011 & 0.016 & 0.007 \\
\hline$R_{\min }$ & 8 & 9 & 10 & 11 & 12 & 13 \\
\hline$R_{\max }$ & 9 & 10 & 11 & 12 & 13 & 14 \\
\hline \hline
\end{tabular}

Similarly, for the $\Gamma$-randomness parameter the results are: 


\begin{tabular}{||c|c|c|c|c||}
\hline \hline Fraction & 0.886 & 0.094 & 0.015 & 0.0039 \\
\hline$R_{\min }$ & 1 & 2 & 3 & 4 \\
\hline$R_{\max }$ & 2 & 3 & 4 & 5 \\
\hline \hline
\end{tabular}

The randomness of the set $\left\{2^{i}\right\}$ in $\mathbb{Z}_{n}$ is larger than in $\Gamma(n)$, due to the irregularity of the subset $\Gamma(n)$ in $\mathbb{Z}_{n}$ (for most $n$ ).

\section{$9 \quad$ Arc lengths distribution}

Consider the random independent choices of $T$ different elements for the $m$-elements "discrete circle" $\mathbb{Z}_{m}=\mathbb{Z} / m \mathbb{Z}$.

Theorem 12. The probability $p_{i}$ of the arclength $k$ occurrence in the subdivision of $\mathbb{Z}_{m}$ by these $T$ points equals to

$$
p_{i}=C_{m-k-1}^{T-2} / C_{m-1}^{T-1}
$$

(and is thus proportional, for a fixed points number $T$, to the elements of the Pascal triangle line, parallel to its side and situated at the distance $T-2$ from the side).

Remark. This "probability" is counting the frequency of the arclength $k$ rather than that of the subdivision containing such arcs: each arc contributes 1 in the counting, independently on the presence of any other arc of the same length in the subdivision containing it.

Proof. Count the length $k$ "initial arcs" of the subdivisions starting at the point 0 (and the "initial arc" being $(0, k))$. The subdivision into $T$ parts, containing this initial arc, are defined by the remaining $T-2$ division points, which have to be chosen from the $m-k-1$ interior integral points of the remaining arc of length $m-k$. Thus the subdivision number equals to the combinations number $C_{m-k-1}^{T-2}$, where $1 \leq k \leq m-T+1$.

The total number of the subdivisions starting at the division point 0 being equal to the combinations number $C_{m-1}^{T-1}$, the probability $p_{k}$ is the ratio of these two combinations numbers (the initial point 0 being irrelevant), which is the Theorem statement.

The probability sum $\sum p_{k}$ is equal to 1 (accordingly to the identity, defining the Pascal triangle), confirming that we had counted every arc just once in our counting of the arcs of length $k$. 


\section{$10 \quad T$-good moduli values}

Definition. An integer $n$ is $T$-good if $T$ is a period of the geometrical progression $\left\{2^{t}(\bmod n)\right\}$, that is if

$$
2^{T} \equiv 1(\bmod n)
$$

The period $T$ is not supposed here to be the minimal period. The same number $n$ is also $k T$-good for any integer multiple of $T$.

The set of the $T$-good numbers has the following "anti-ideal" property: it contains together with any integer all its divisors (since the divisibility by $n$ implies the divisibility by its divisors).

Theorem 13. The set of T-good numbers contains together with any two its elements their minimal common multiple.

Proof. The number $2^{T}-1$, divisible by both elements, is also divisible by their minimal common multiple.

The following properties of the $T$-good values set are evident by the very definition.

1. The number $2^{T}-1$ is $T$-good.

2. The $T$-good numbers are its divisors, like $\{1,3,5,15,17,51,85,255\}$ for $T=8$.

In other words:

Theorem 14. The minimal common multiple of the $T$-good numbers is equal to $2^{T}-1$.

\section{References}

[1] Arnold, V. I., Dynamical System of Fermat-Euler and statistics of the arithmetics of geometric progression. Functional Analysis and its applications, 2003, vol. $37 \mathrm{~N} \mathrm{1,} \mathrm{pp} \mathrm{1-20.}$

[2] Arnold, V. I., Weak asymptotics of the numbers of the solutions of diophantine problems. Functional Analysis and its Applications, 1999, vol. 33 N 3, pp. 65-66. 